
\title{
3 Research Suare \\ Percutaneous Radiofrequency Rhizotomy for Prompt Pain Management in Peculiar Cases of Trigeminal Neuralgia
}

Nadya Zaragita

National Brain Center MT Haryono Kav. 11

Mustaqim Prasetya

National Brain Center MT Haryono Kav. 11

Adi Sulistyanto ( $\square$ adisulistyanto.nbc@gmail.com )

National Brain Center MT Haryono Kav. 11

\section{Case report}

Keywords: trigeminal neuralgia, percutaneous radiofrequency rhizotomy, brain tumor, secondary trigeminal neuralgia, minimally invasive, trigeminal schwannoma, epidermoid cyst

Posted Date: March 3rd, 2021

DOI: https://doi.org/10.21203/rs.3.rs-212175/v1

License: (c) (i) This work is licensed under a Creative Commons Attribution 4.0 International License. Read Full License 


\section{Abstract}

Background: Trigeminal neuralgia is a neurological condition characterized by episodes of intense stabbing or electric shock-like pain caused by disorder of the fifth cranial nerve. Most TN cases are caused by vascular compression, but rarely it can be caused by tumor or autoimmune diseases (e.g. multiple sclerosis).

Case presentation: We report two rare cases of secondary trigeminal neuralgia caused by tumors. The $1^{\text {st }}$ patient is a 38 year old male with intractable pain and MRI result suggestive of trigeminal schwannoma at the left cerebellopontine region, while the $2^{\text {nd }}$ patient is a pregnant 26 year old female with MRI result suggestive of an epidermoid cyst at left cerebellopontine region compressing the trigeminal and and facial nerve. Surgical option was declined by both patients because of the associated risks although immediate pain relief was needed. Percutaneous radiofrequency rhizotomy (PRFR) was chosen for prompt pain management and results showed immediate pain relief.

Conclusion: We conclude that PRFR is an effective option for pain management in secondary trigeminal neuralgia patient in whom primary treatment for the underlying cause is not feasible.

\section{Background}

Trigeminal neuralgia is a neurological condition characterized by episodes of intense stabbing or electric shock-like pain caused by irritation of the fifth cranial nerve (trigeminal nerve). International Headache Society divided trigeminal neuralgia into three groups: primary (classic), idiopathic, and secondary. Classic trigeminal neuralgia is when the condition is caused by vascular compression, while secondary trigeminal neuralgia have other underlying causes, such as tumors and autoimmune diseases. ${ }^{[1]}$ Treatments of trigeminal neuralgia vary, including drugs, percutaneous rhizotomy (balloon; radiofrequency; glycerol), gamma knife surgery, and microvascular decompression. ${ }^{[2]}$ When quality of life is still compromised or when pain is intractable with drugs, other intervention may be necessary. Microvascular decompression has been the mainstay procedure for trigeminal neuralgia; however, not all patients have the access and are the candidate for this procedure.

We report two cases of secondary trigeminal neuralgia who declined or were unable to undergo surgery for the underlying cause which is tumor on the trigeminal nerve. After thorough discussion, PRFR was chosen for both patients. Patients were sedated during the beginning of procedure. Fluoroscopy was used as a guidance to aim needle to the foramen ovale. Patient was later woken up and stimulated to confirm correct placement of the needle on the desired trigeminal branch. Sedation was then established once again until rhizotomy was completed. PRFR was done using Cosman ${ }^{\mathrm{TM}} \rrbracket$ Radiofrequency with heat of $60-80^{\circ} \mathrm{C}$ for as long as $60-90$ seconds.

\section{Case 1}


A 38-year-old man presented to the clinic with chief complaint of excruciating pain at the left side of his face, especially the forehead area for the last 1 month. Pain appeared about 10 times a day every single day, with Numeric Index Pain Scale (NIPS) of 10 and Barrow Neurological Institute Pain Scale (BNIPS) of 4. His pain remained intractable on medications and the patient demanded some form of immediate pain relief as the trigeminal neuralgia episodes disturbed his quality of life. However, he refused surgery due to the associated risks. Medications were no longer preferable since patient experienced epigastric pain and nausea due to medication side effects and was repeatedly admitted to the emergency department over several months due to this complaint.

\section{MRI}

MRI results showed a singular mass at the left cerebellopontine (CPA) region extending towards the Meckel's cave was found. The mass appeared to enhance homogenously after contrast administration, suggestive for trigeminal schwannoma (Figs. 1 and 2).

\section{Management}

After thorough discussion, we decided to perform percutaneous radiofrequency rhizotomy (PRFR) for immediate pain relief (Fig. 3), followed by gamma knife surgery for tumor control. Gamma knife was chosen to be performed after PRFR due to its gradual effect, which may take up until 6 months until tumor size reduction.

\section{Result}

The pain persisted until the fourth day after PRFR was done, but then diminished completely (BNIPS I, NIPS 0), with slight facial numbness at the left side. After one-month follow-up, pain reappeared with the same frequency but much lesser intensity (NIPS 5-6). The patient was prescribed gabapentin $300 \mathrm{mg}$ once a day and carbamazepine $200 \mathrm{mg}$ once a day. Patient was scheduled for gamma knife surgery for the tumor, which was performed in another center 3 months after PRFR. The patient reported complete pain relief (BNIPS I, NIPS 0) during the 6-month follow up.

\section{Case 2}

A 26-year-old woman presented to our clinic with episodes of intense pain at her left cheek. For the last 5 years, the pain appeared three times a day every single day, with a reported severity of NIPS 9 and BNIPS IV. The pain episodes disturbed all the patient's activities and greatly affected her quality of life. To achieve adequate pain relief, the patient had to take Oxcarbazepine $300 \mathrm{mg}$ twice a day; yet the patient was also 8-week pregnant and the available safe drugs for pain relief did not give the desired effect. She wanted other modality for pain relief that was safe for her pregnancy.

\section{MRI}


MRI results showed a thin-walled cystic lesion at the left CPA region with size of $1.3 \times 2.8 \times 1.4 \mathrm{~cm}$, which compressed the trigeminal nerve and the facial nerve (Figs. 4 and 5). In T1 with contrast, mass did not appear to be enhanced, suggestive for epidermoid cyst.

\section{Management}

Pain relief drugs that are considered safe for pregnancy such as ibuprofen and paracetamol were not effective during the neuralgia episodes. Commonly prescribed drugs for trigeminal neuralgia, such as gabapentin, phenytoin, or carbamazepine were not safe especially before the second trimester. Surgery was not considered safe either for the first trimester of pregnancy. The patient agreed to undergo PRFR procedure for immediate pain relief as it was a modality considered most safe yet effective for her condition. During the procedure, the patient wore an anti-radiation apron to ensure protection.

\section{Result}

Soon after the procedure, patient reported complete relief of pain (Barrow Neurological Institute Pain Scale I, Numeric Index Pain Scale 0) and slight left sided facial numbness. After 1 week of procedure, pain appeared once for 1 hour and a NIPS scale of 0 , but complete pain relief has been maintained since then. Facial numbness gradually improved after PRFR. At 6-month follow up, the patient remained painfree without use of any pain-relieving medications.

\section{Discussion}

There are many options of medical treatments for trigeminal neuralgia, but the choice of treatment has to be individualized for each patient. When pain is not adequately controlled, quality of life is compromised and psychological problem such as depression can occur. ${ }^{[3]}$ In our cases, both patients had secondary trigeminal neuralgia which was caused by tumor lesion and tumor removal remained as definitive treatment. However, both patients had unfavorable conditions for surgery or declined open neurosurgical procedure, rendering tumor removal unfeasible.

Microvascular decompression (MVD) was proven effective in most trigeminal neuralgia cases, but prior studies proved that in 3-20\% patients required re-treatment after $2-3$ pain-free years. ${ }^{[4,5]}$ It remained the definitive therapy for primary trigeminal neuralgia including cases where medications are no longer satisfactory. However, this procedure carries some risks, including facial numbness, deafness, facial palsy, CSF leak, meningitis, and death, with total periprocedural complication rate as high as $19.3 \%$. ${ }^{[6]}$ Secondary trigeminal neuralgia caused by tumor treated with open surgery for removal showed immediate pain relief without neurologic deficit. ${ }^{[7]}$

Percutaneous radiofrequency rhizotomy is another form of modality to treat trigeminal neuralgia. Indications include bilateral trigeminal neuralgia, elderly patients, recurrence after failed MVD, vertebrobasilar dolichoectasia and multiple sclerosis. ${ }^{[3]}$ Initial pain-relief effect reach $95 \%$, but over half of 
patients who had rhizotomy needed re-treatment after pain-free period of $3-4$ years. ${ }^{[3,5]}$ This procedure is most commonly done compared to other options due to the feasibility and is relatively easier compared to open surgery as it is minimally invasive. Overall, recurrence rate after 6 months of all trigeminal neuralgia cases reached $13.5 \%$ and most often occur along the V1 distribution. For secondary trigeminal neuralgia, reoccurrence was as much as $37.5 \%$. ${ }^{[8]}$

Gamma knife surgery is one of the available options for trigeminal neuralgia treatment, however, painrelief effect is not immediate and may take approximately 1-3 weeks after the procedure. This treatment is least invasive and good for patients with co-morbidities, high-risk medical illness, or refractory pain after prior procedures. However Gamma Knife may not be available in many medical centers. In Indonesia, there are only two centers with gamma knife facilities. Furthermore the cost of treatment is outside the coverage of the national health insurance which is the sole health insurance of about $80 \%$ of Indonesian people.

Based on all the available modalities in our center, authors and patients decided to choose the most effective personalized treatment for patients' condition. Quality of life improved greatly and patient could resume the daily activity for a short period of time before continuing to the definitive treatment. PRFR can be used as an alternative to alleviate pain in situations where surgical procedures or medications are unlikely for short period of time.

\section{Conclusion}

Our findings show that although lesion removal in secondary trigeminal neuralgia was not undertaken due to unfavorable circumstances, the PRFR procedure resulted in good outcome for dire pain management. We conclude that PRFR prior to surgical procedure can be an alternative procedure for immediate pain alleviation compared to other procedures, or in cases where immediate surgery is not possible.

\section{Declarations}

\section{Ethics approval and consent to participate}

Institutional ethics approval was waived for this study. The authors certify that they have obtained all appropriate patient consent forms and both patients consented to participate in this study.

\section{Consent to participate}

The authors certify that patient(s) has/have given his/her/their consent for his/her/their images and other clinical information to be reported in the journal. The patients understand that their names and initials will not be published and due efforts will be made to conceal their identity, but anonymity cannot be guaranteed. 
Availability of data and materials

The dataset analyzed in the current study are not publicly available due to institutional confidentiality policies, but may be available from the corresponding author on reasonable request.

\section{Competing interest}

The authors declare no competing interests

\section{Funding}

No funding was obtained for this study

\section{Authors contributions}

NZ, MP, and AS analyzed and interpreted patient data regarding PRFR and secondary trigeminal neuralgia. MP and AS performed the procedures and gave major contribution in writing the manuscript. NZ reviewed literature and drafted the final version of the manuscript. All authors read and approved the final manuscript.

\section{Acknowledgments}

All authors would like to thank Indonesia National Brain Center and its Neurosurgery Department for the support lended towards the completion of this study.

\section{References}

1. Vincent M, Wang S. Headache Classification Committee of the International Headache Society (IHS) The International Classification of Headache Disorders, 3rd edition. Cephalalgia 2018;38:1-211.

2. Obermann M. Treatment options in trigeminal neuralgia. Ther Adv Neurol Disord 2010;3:107-15.

3. Yadav YR, Nishtha Y, Sonjjay P, Vijay P, Shailendra R, Yatin K. Trigeminal Neuralgia. Asian J Neurosurg 2017;12:29682044.

4. R.O. S-M, M. L, J.S. C, J. C, M.M. W, N.M. B. Recurrent or refractory trigeminal neuralgia after microvascular decompression, radiofrequency ablation, or radiosurgery. Neurosurg Focus [Internet] 2005;18:e12. Available from: http://www.embase.com/search/results?

subaction=viewrecord\&from $=$ export\&id $=\mathrm{L} 43730024$

5. Hitchon PW, Holland M, Noeller J, Smith MC, Moritani T, Jerath N, et al. Options in treating trigeminal neuralgia: Experience with 195 patients. Clin Neurol Neurosurg [Internet] 2016;149:166-70. Available from: http://dx.doi.org/10.1016/j.clineuro.2016.08.016

6. Fouad W. Management of trigeminal neuralgia by radiofrequency thermocoagulation. Alexandria $\mathrm{J}$ Med [Internet] 2011;47:79-86. Available from: http://dx.doi.org/10.1016/j.ajme.2011.02.001 
7. Son DW, Choi $\mathrm{CH}$, Cha SH. Epidermoid tumors in the cerebellopontine angle presenting with trigeminal neuralgia. J Korean Neurosurg Soc 2010;47:271-7.

8. Emril DR, Ho KY. Treatment of trigeminal neuralgia: Role of radiofrequency ablation. J Pain Res 2010;3:249-54.

\section{Figures}

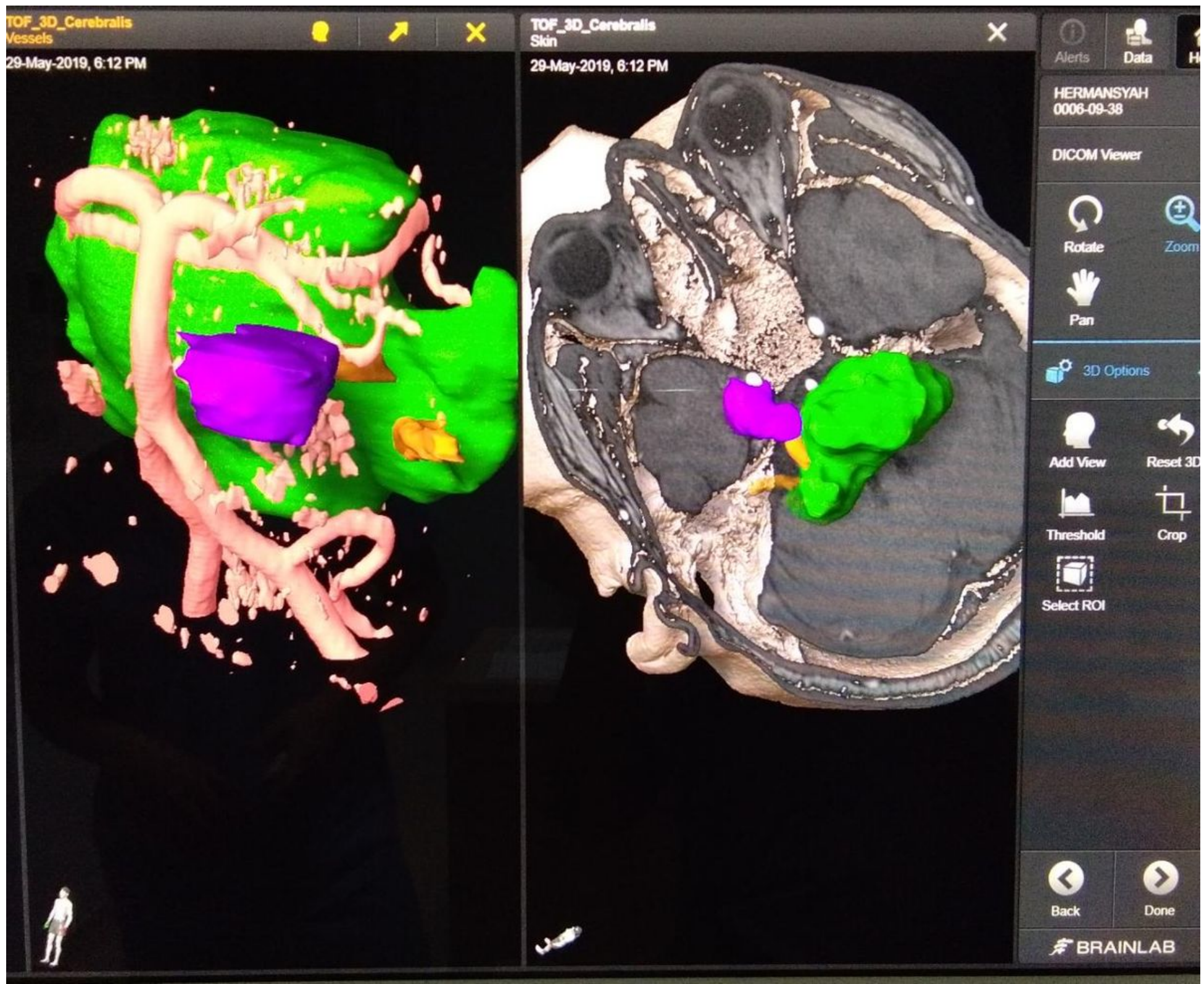

Figure 1

Three-dimensional reconstruction of brainstem using Brainlab ${ }^{\text {TM }}$ software, vertebrobasilar arteries, left trigeminal nerve, and a mass extending from the prepontine cistern to the left Meckel's cave. 


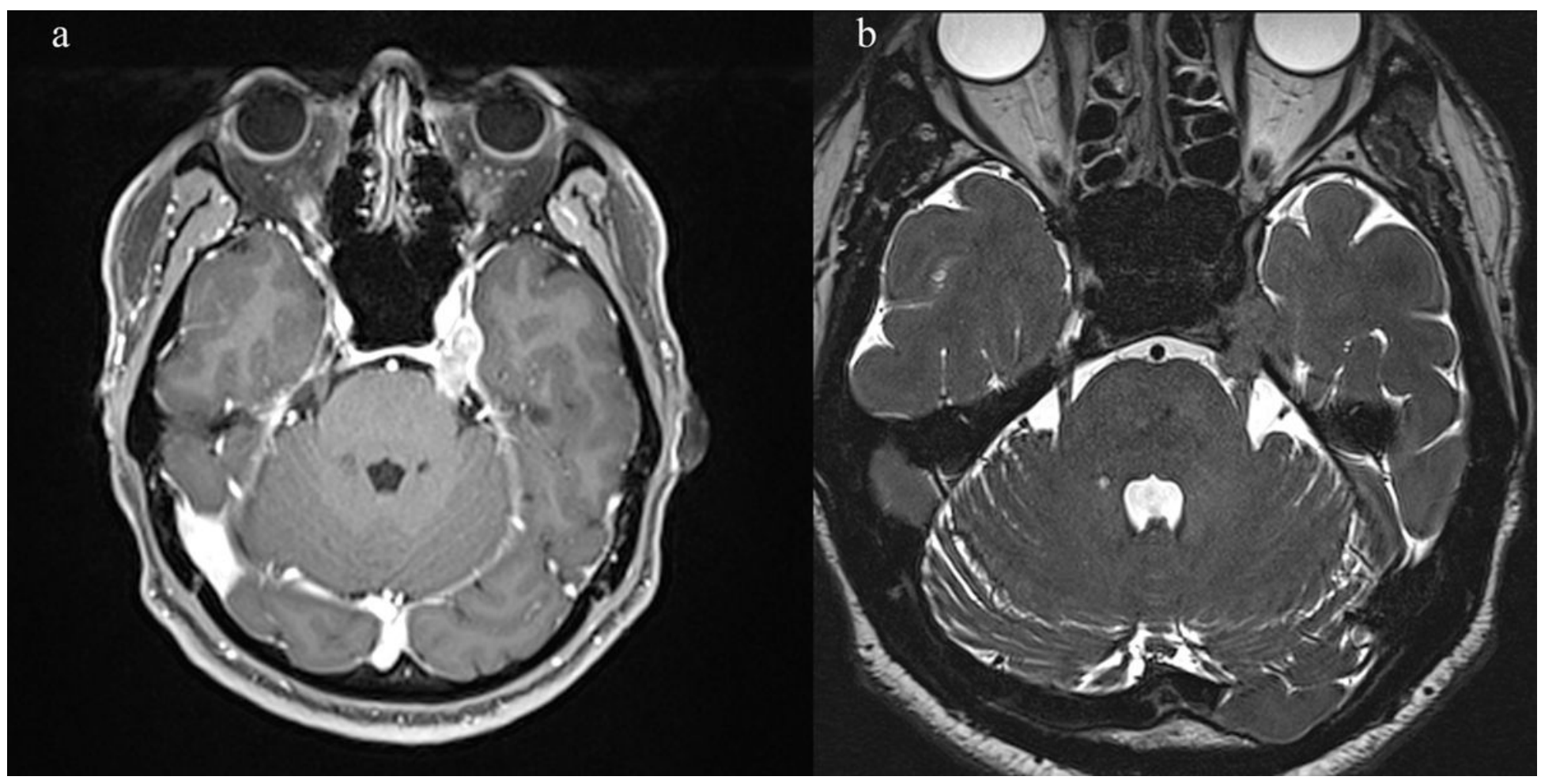

Figure 2

(a) T1 with contrast enhancement showed hyperintense mass and (b) T2 sequence showed isointense mass at the left CPA. 


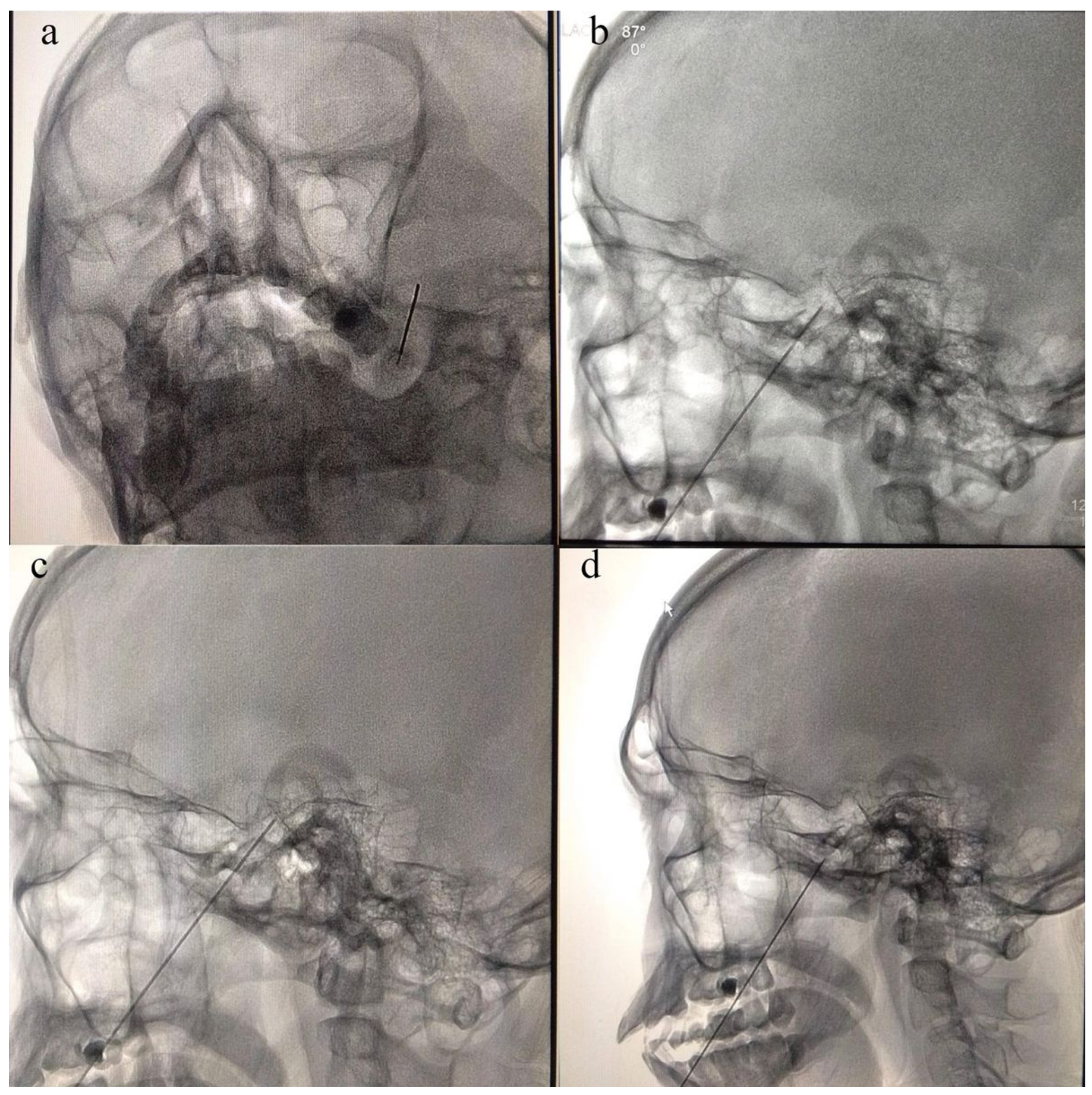

Figure 3

(a) Needle targeted to Gasserian ganglion above foramen ovale, (b) position at V1, (c) V2, and (d) V3 confirmed by stimulation 


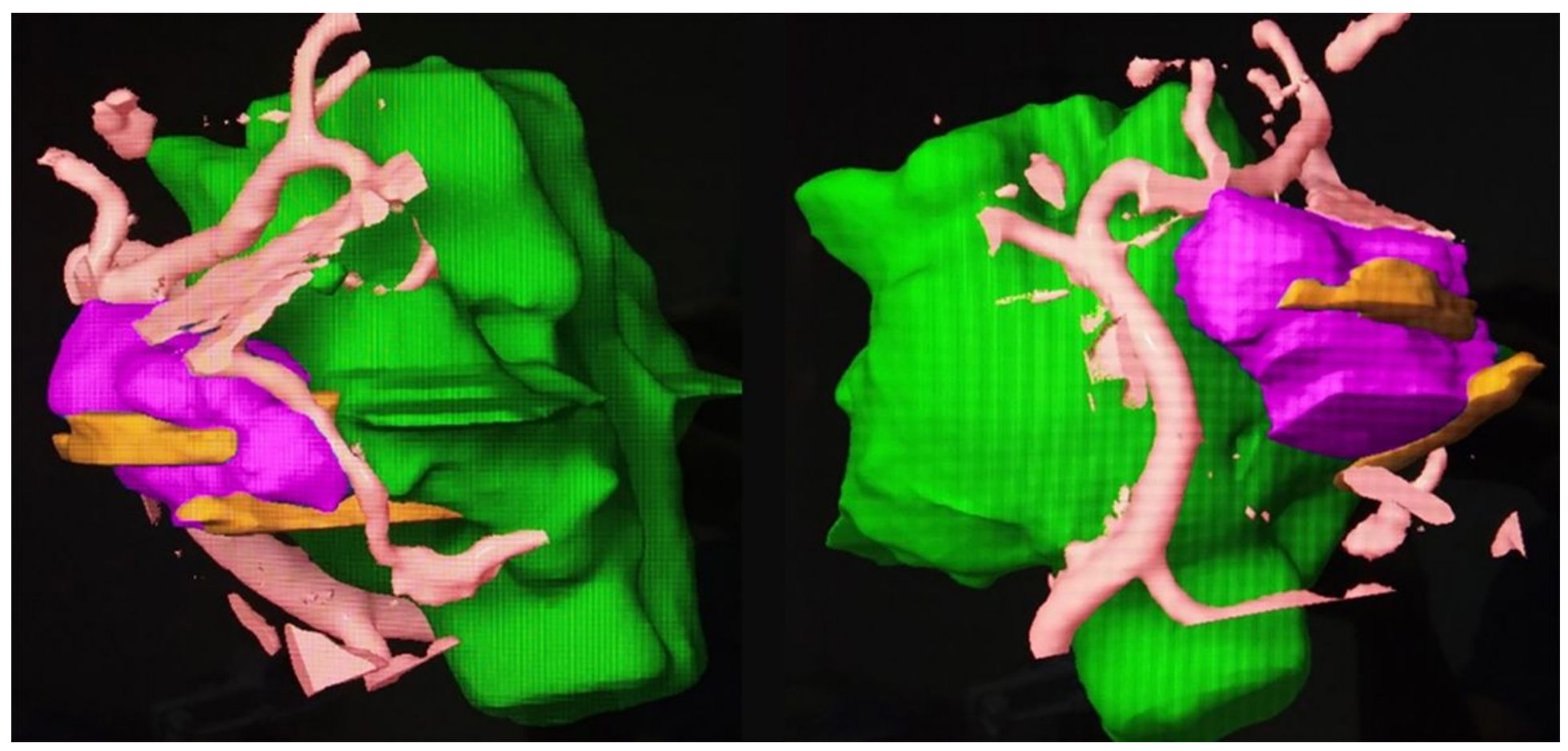

Figure 4

Three-dimensional reconstruction of brainstem, posterior circulation, and a mass that compresses the left trigeminal nerve and facial nerve.

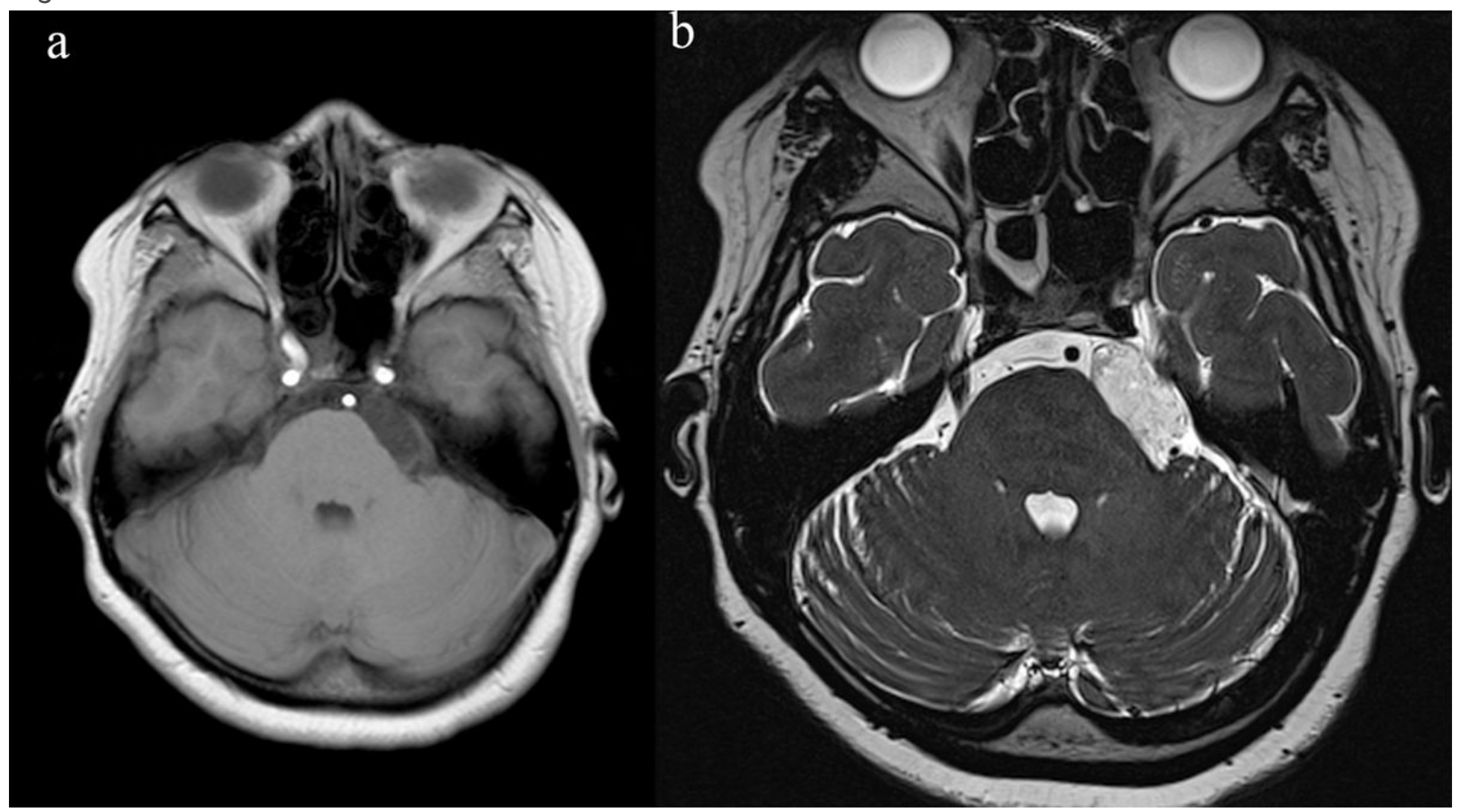

Figure 5 
(a) T1 with contrast enhancement and (b) T2 sequence of the patient. Size of the lesion was $1.3 \times 2.8 \times 1.4$ $\mathrm{cm}$ located at the left CPA region.

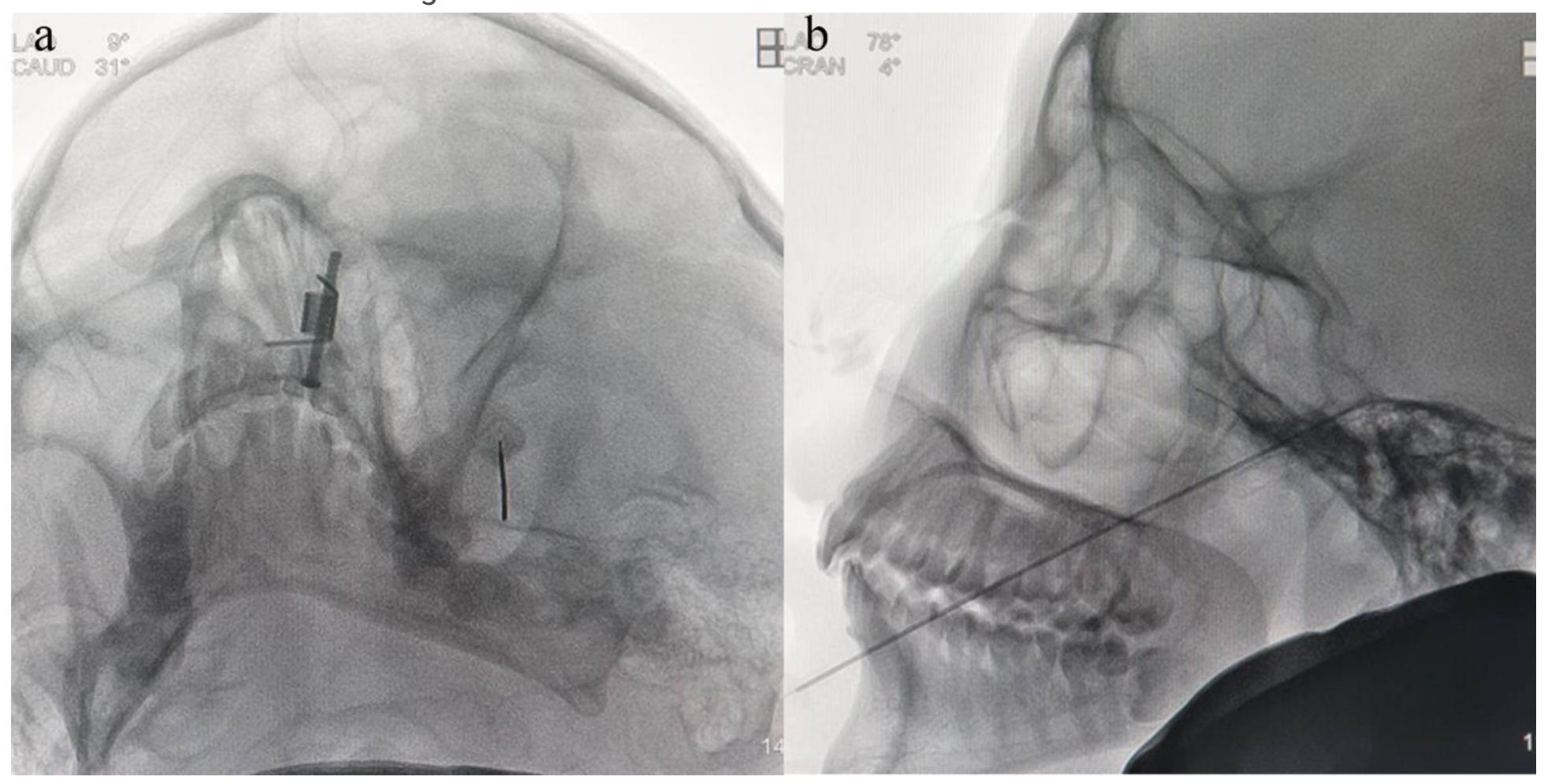

\section{Figure 6}

(a) and (b) PRFR needle targeted to the left foramen ovale. 